Thomas Abel ${ }^{1}$, Esther Walter ${ }^{1}$, Steffen Niemann ${ }^{1}$, Rolf Weitkunat ${ }^{2}$

1 Unit for Health Research, ISPM, University of Berne

${ }^{2}$ IBE, Klinikum Grosshadern, Ludwig Maximilians University, Munich

\section{The Berne-Munich Lifestyle Panel}

Background and baseline results from a longitudinal health lifestyle survey

\title{
Summary
}

The Berne-Munich Lifestyle Panel (BMLP) studies health relevant lifestyles among some 2000 adults in Switzerland and Germany. This paper introduces the theoretical background and empirical concept of the BMLP. Sociological theory provided the guidelines for the development of an empirical model that measures structures and dynamics of health fifestyes. Health lifestyles are explained as the product of the complex interplay between health related behaviours, orientations and social resources. Residents of Berne (Switzerland) and Munich (Germany) in the age between 55 and 65 years were contacted in 12 months periods and interviewed by telephone (CATI). The questionnaire comprised some 200 questions on selected aspects of health lifestyles and health status. Interviews were conducted in two waves in Munich (1996 and 1997) and three waves in Berne (1996/97/98). The paper reports findings from baseline data analysis and explores cultural differentiations with respect to the distribution of 1 health relevant behaviours, orientations and social resources, 2. triggers of lifestyle change (life events), 3, mediating factors (Health Locus of Control, Sense of Coherence). Initial results from the search for patterns of health behaviours are also reported. The findings show considerable differences but also impressive similarities in health lifestyle elements across the two samples. There is also preliminary evidence for meaningful patterns of health behaviours in the cohort under investigation. Moreover, the findings clearly demonstrate the need for a gender specific approach in the analysis of cultural differences in health behaviours and lifestyles.

Since the late 1960ies the lifestyle approach has become increasingly popular in health research. In its early stages of application its major contribution was to draw attention to the behavioural aspects of health and risk of disease. With respect to methodological advancements the lifestyle approach has strengthened the development of multivariate models measuring the more complex links between various risk behaviours and health. Since the 1980ies; however, the lifestyle approach in health research has been criticised for its shortcomings and pitfalls, particularly when it is used in a rather narrow risk-factortype conception. Indeed, until today many so called "lifestyle" studies are little more than risk behaviour studies often stressing individual behaviour while overlooking structural factors. Such and other obvious limitations can be explained by an apparent lack of theory and a restricted, discipline-bound perspective, typical for many earlier approaches.

More recently, attempts have been made to overcome these limitations and to develop more comprehensive health lifestyle models ${ }^{1,2}$. In line with sociological lifestyle theories and compatible with the Public Health paradigm, one can argue that lifestyle research should be focused on populations and distinct sub-groups rather than on individuals. Also, an interdisciplinary perspective is needed that accounts for the bio-psycho-social factors and their complex links. In order to overcome the limitations of a restricted perspective on health risk, salutogenic approaches to health lifestyles including the factors that promote or maintain good health, also need to be developed. In the present paper the BerneMunich Lifestyle Panel study (BMLP) is introduced. This study 
explores health behaviours in a comprehensive lifestyle perspective and offers a new approach towards a Public Health based understanding of lifestyle ${ }^{3}$. It applies a sociological framework with reference to Max Weber's sociology of lifestyle, life chances and life conduct and explores empirically the links of structural and behaviour factors of health. Health lifestyles are explained as the product of the complex interplay between health related behaviours, orientations and social resources. Health lifestyles are defined here as interacting patterns of health related behaviours, orientations and resources adapted by groups of individuals in response to their social, cultural and economic environment ${ }^{4,5}$.

In the BMLP the three health lifestyle dimensions and their correlates are measured by some 200 items. A longitudinal study that includes almost 2000 adults has been conducted in Berne (Switzerland, $N=1119$ ) and Munich (Germany, $N=780$ ). In both countries, data are collected by telephone (CATI) applying almost identical questionnaires in both samples. Cross-sectional data provide the grounds for comparative analysis which can be used for testing for cultural specificity and cross-cultural similarities. However, today there is empirical evidence on male-female differences that may intervene or interact with cultural differences in health and health behaviour ${ }^{6,7}$. Consequently, the present study also examines gender differences in the observed cultural variations.

Future analyses of the longitudinal BMLP data will allow to study stability and change of health lifestyle structures over time. The present paper, however, provides the introduction into the conceptual and methodological background of this new approach. Baseline results from the first wave of data collection describe the distribution of health lifestyle factors across the two city samples.

\section{Conceptual Background}

In 1993 Cockerham and his colleagues published a theoretical account of Max Weber's theory of lifestyle and its contributions to our understanding of modern health related lifestyles ${ }^{8}$. One major conclusion that can be drawn from this theoretical analysis is, that lifestyles are the product of a complex interplay between structurally anchored life chances and creative actions or achievements of individuals who act upon given objective and subjective life conditions. Health lifestyles are neither totally determined by social conditions nor purely a matter of individual choices. Rather, within restricting socio-economic conditions and cultural normative borders, individuals have a more or less wide range of choices from a variety of health related behaviours ${ }^{2}$. The development and realisation of health relevant behaviours and orientations depend on the availability of sufficient social resources such as income, time, education. In turn, such resources can positively or negatively be affected by health damaging behaviour patterns. Increased health risk or lower health status are likely to reduce resources and life chances, in the case of HIV risk behaviour even survival chances, and to increase or perpetuate social inequality. The unequal distribution of social resources is at the core of the sociological understanding of health lifestyles as a collective rather than an individual phenomenon ${ }^{4}$.

\section{Research questions and measurement model in the BMLP}

The BMLP project approaches substantive and methodological issues in health lifestyle research. It applies a bio-psycho-social definition of health ${ }^{9}$. Health in its broader definition is considered as an outcome of health relevant lifestyles while particular dimensions of health (physical and psychological functioning) are, at the same time seen as important resources for realising certain health relevant behaviours. With respect to stability over time, we assume that health lifestyles are complex dynamic systems ${ }^{10}$ with an increased probability of change after the occurrence of major life events ${ }^{5}$. We further assume that the effect of life events on health lifestyle patterns will depend on a mediating effect of individual internal resources. In the present study "Health Locus of Control" 14 and "Sense of Coherence" 15 are included as examples for such internal resources. Longitudinal comparative data are collected to perform five tasks: 1. Selection and development of meaningful health lifestyle indicators and descriptive analysis of their distribution in a defined population. 2. Analysis of the links between health lifestyle elements within and between the three dimensions of behaviours, orientations and resources. 3. Identification of distinct health lifestyle groups based on patterns of health related behaviours, orientations and resources. 4. Analysis of stability and change of health lifestyles over time and across two city populations in two European nations. 5. Explorations into selected crossnational differences and gender variations.

The present paper reports baseline findings on tasks 1,2 and 5. Analyses on tasks 3 and 4 are currently underway.

Figure 1 presents the measurement model of the BMLP.

\section{Data and Methods}

The BMLP is a study of health lifestyles in a specified age cohort. The 


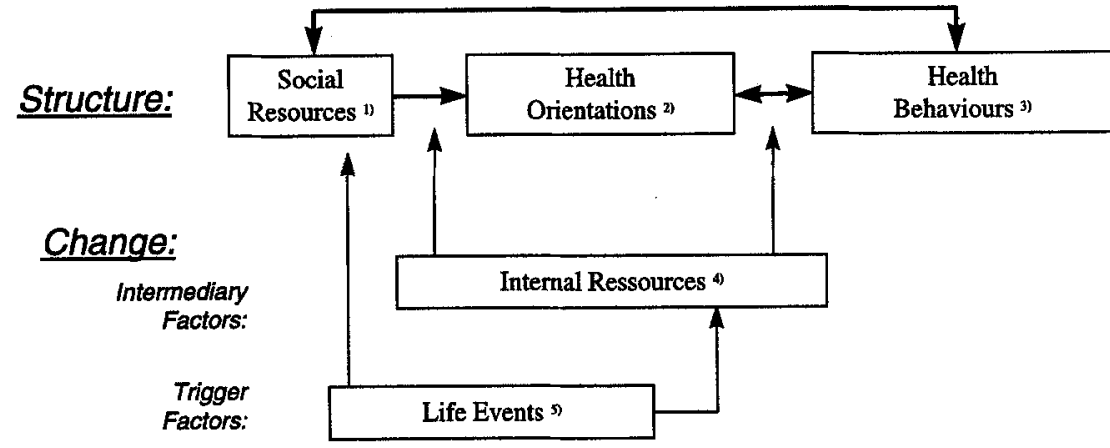

1) Financial resources, education, occupational prestige, social support, etc.

2) Trust in modern and alternative medicine, rationality orientations, concern about eating, religious beliefs, etc.

3) Smoking, alcohol consumption, physical activity, eating habits, set belt use, etc.

4) Health Locus of Control, Sense of Coherence.

5) Major Life Events.

Figure 1. Measurement model in the BMLP.

age group of 55 to 65 years was chosen for three reasons. First, from a Public Health and Health Promotion perspective, adults in transition between active labour and retirement status present an important, yet "under-researched" population. Second, we expect patterns of health behaviours and orientations in that later stage of life to be more consistent, stable and therefore more clearly detectable. And third, major life events such as loss of a spouse, major ill- ness, unemployment etc. are more likely to occur in this age group, providing sufficient numbers of cases to explore the effect of life events on the dynamics of health lifestyles.

Two random samples were drawn from official registration lists of German speaking inhabitants in the municipalities of Berne (Switzerland) and Munich (Germany). Sample sizes were $\mathrm{N}=1913$ in Berne and $N=1526$ in Munich. Interviews were conducted by tele- phone (CATI) and yielded net completion rates in Berne of $64.4 \%(\mathrm{~N}=1119)$ and in Munich of $57.7 \%(\mathrm{~N}=780)$ in the first wave. The second wave yielded $82.9 \%$ $(\mathrm{N}=924$, Berne) and $75.4 \%$ ( $\mathrm{N}=595$, Munich) net completion rates. In Berne a third wave yielded a net completion rate of $92 \%$ $(\mathrm{N}=839)$. The questionnaire comprised some 200 questions on health lifestyles, health status and sociodemographic factors with mainly closed answer categories. Questionnaire items where selected to measure three health lifestyle dimensions (behaviours, orientations, social resources), triggers of lifestyle change (life events), intermediary factors (Health Locus of Control and Sense of Coherence) assumed to modify the effects of the triggers on the lifestyle structure. Wherever possible, lifestyle elements and their correlates were assessed using pre-tested items or scales.

This paper presents data from the first wave. The following health lifestyle elements were selected for the analyses (see appendix for more details on the variables).

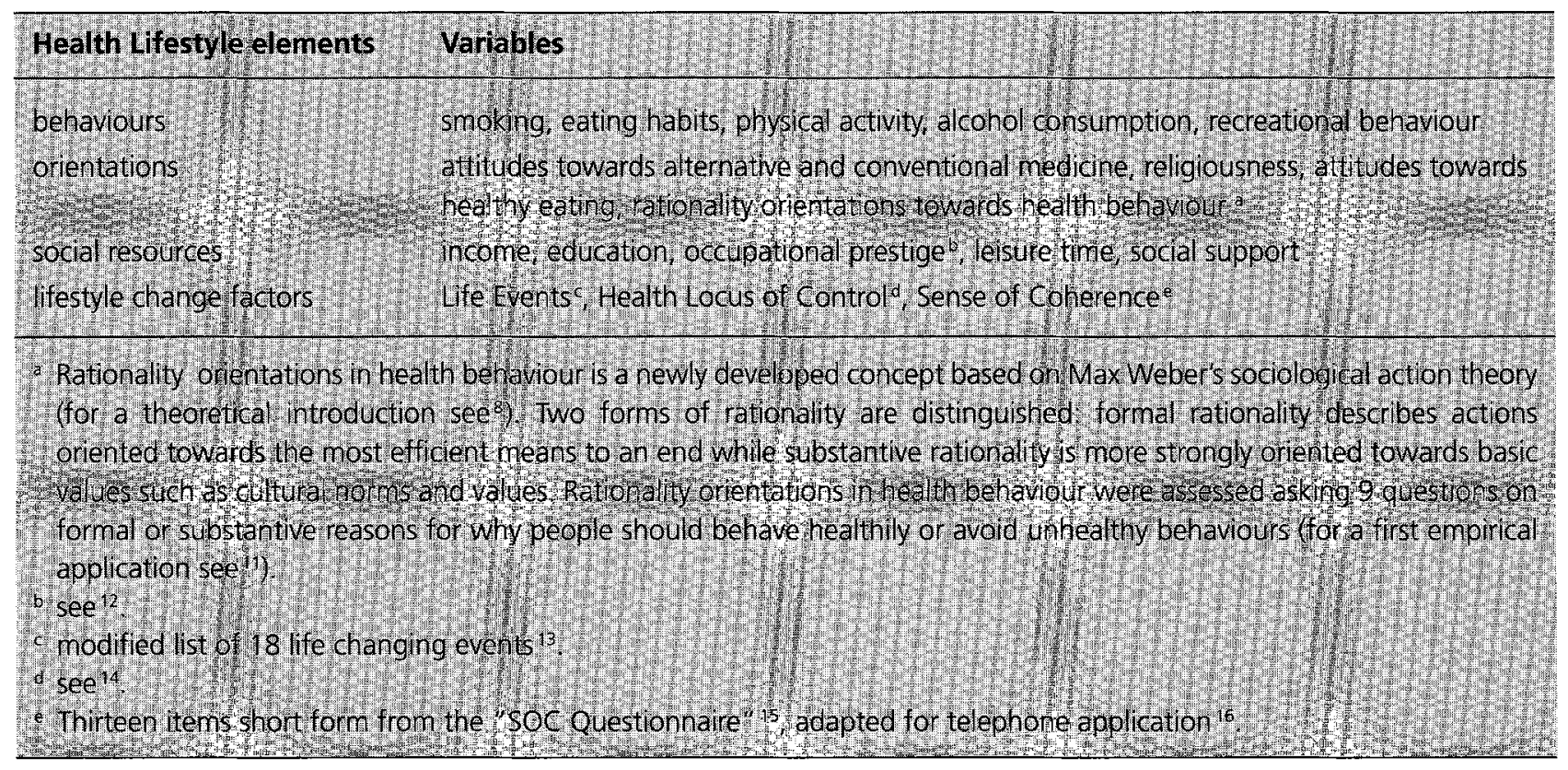


The BMLP questionnaire also includes some 80 items to study the physiological and psychological dimensions of health. Concepts and empirical findings of measuring health status in the BMLP will be published separately.

Basic descriptive statistics were utilised for baseline analyses. Findings on the general distribution of health lifestyle elements in the two study populations are presented here. Data were analysed by first constructing comparable answer categories, then testing for cross-cultural differences using contingency tables. Cross-cultural differences were further explored for their gender specific nature.

\section{Empirical Findings}

Tables 1-4 present two kinds of results from contingency table analyses of lifestyle data in the two city samples. First, comparisons between the two samples are displayed. Second, cultural differences and similarities are examined for possible gender effects. Table 5 lists in a summary form which associations in Tables 1-4 showed statistically significant cultural differences, for the whole sample and for both gender groups separately. Tables $6,6 \mathrm{a}$ and $6 \mathrm{~b}$ finally, display correlation coefficients corresponding to bi-variate patterns among health lifestyle behaviours.

\section{Behaviours}

Table 1 presents findings for prevalences of health lifestyle behaviours. Smoking behaviour is measured in a three-category coding scheme (non smoking or less than one cigarette a day; one to ten cigarettes a day; more than 10 ). Comparing these categories we find a striking similarity in Berne and Munich. The proportion of non, light, and heavy smokers are almost the same in Berne $(78.9 \% ; 7.8 \%$; $13.3 \%)$ and Munich $(79.9 \% ; 7.7 \%$;
$12.5 \%$ ). This cross cultural similarity is observed for both genders.

With respect to beer drinking, based on three categories of consumption, we find in Berne significantly more non consumers of beer and in Munich higher rates of daily beer drinking $(p<0.001)$. Testing for cultural differences in men and women separately underlines this pattern. Among men about $60 \%$ in Berne and about $78 \%$ in Munich consume beer $(\mathrm{p}<0.001)$; respective numbers for women are $15 \%$ in Berne and $45 \%$ in Munich $(p<0.001)$. Conversely to beer consumption, wine drinking is more frequent in Berne than in Munich $(\mathrm{p}<0.05)$. However, an assumption about a general cultural effect referring to the well known "beer culture" in Bavaria and the tradition of wine production and consumption in Switzerland is not supported by our data. Exploring gender differences Table 1 shows that women in Berne and in Munich show highly similar wine consumption patterns. Consequently the culture effect in wine consumption seems to apply among men only $(\mathrm{p}<0.001)$.

Fruits, salads, potatoes and vegetables are included here for their role as the main sources of vitamin $\mathrm{C}$ in the general population in Switzerland and Germany. Bernese men and women eat more of these food stuffs but also consume more meat than their Munich counterparts $(\mathrm{p}<0.001)$. Sausage and ham consumption is significantly higher in our Munich sample $(p<0.001)$. These results on the cultural differences in eating hold for both genders.

Concerning physical activity we find strong similarities in our Bernese and Munich samples: no sports (Berne: 36.8\%; Munich: $37.3 \%$ ), two hours or less per week (Berne: 28.5\%; Munich: 27.8\%) and three hours or more (Berne: $34.7 \%$; Munich: $34.9 \%$ ). The similarities in physical activity are found for both genders.
Seat belt use is reported high in Munich $(95.1 \%$ always or very often) as well as in Berne (90.4\%). However, in Berne the proportion of respondents in the category seldom/never $(6.3 \%)$ is almost twice as high as in Munich (3.5\%). While Bernese men are more likely not to use seat belts compared to their Munich counterparts $(\mathrm{p}<0.01)$, this difference is smaller and statistically not significant between Bernese and Munich women.

Based on three categories (often/ very often; some times, rarely/ never) going for walks is significantly more frequent in Munich than in Berne $(p<0.01)$. However, this difference is only found among men $(p<0.05)$ yet not among women.

With respect to health lifestyle behaviours overall, we find strong similarities in the distribution of smoking and physical activity in Berne and Munich. While other health behaviours show considerable cross-cultural variations, we find no indication for a singular trend or consistency with respect to more healthy or unhealthy behaviours typical in one or the other city population. Gender specificity in cultural differences in health behaviour is considerable. Wherever cultural differences are observed for only one of the two sexes, they occurred among men. While women in Berne and in Munich differ significantly only with respect to beer consumption and eating habits, men show additionally cultural variation in wine consumption, seat belt use and going for walks. Table 5 presents an overview of the observed cultural variations for the whole sample and separately for the two genders.

\section{Orientations}

For comparative analysis substantive and formal rationality orientations towards health behaviour were coded into two sub-categories, measuring agreement versus dis-agreement or indifference. A majority in 


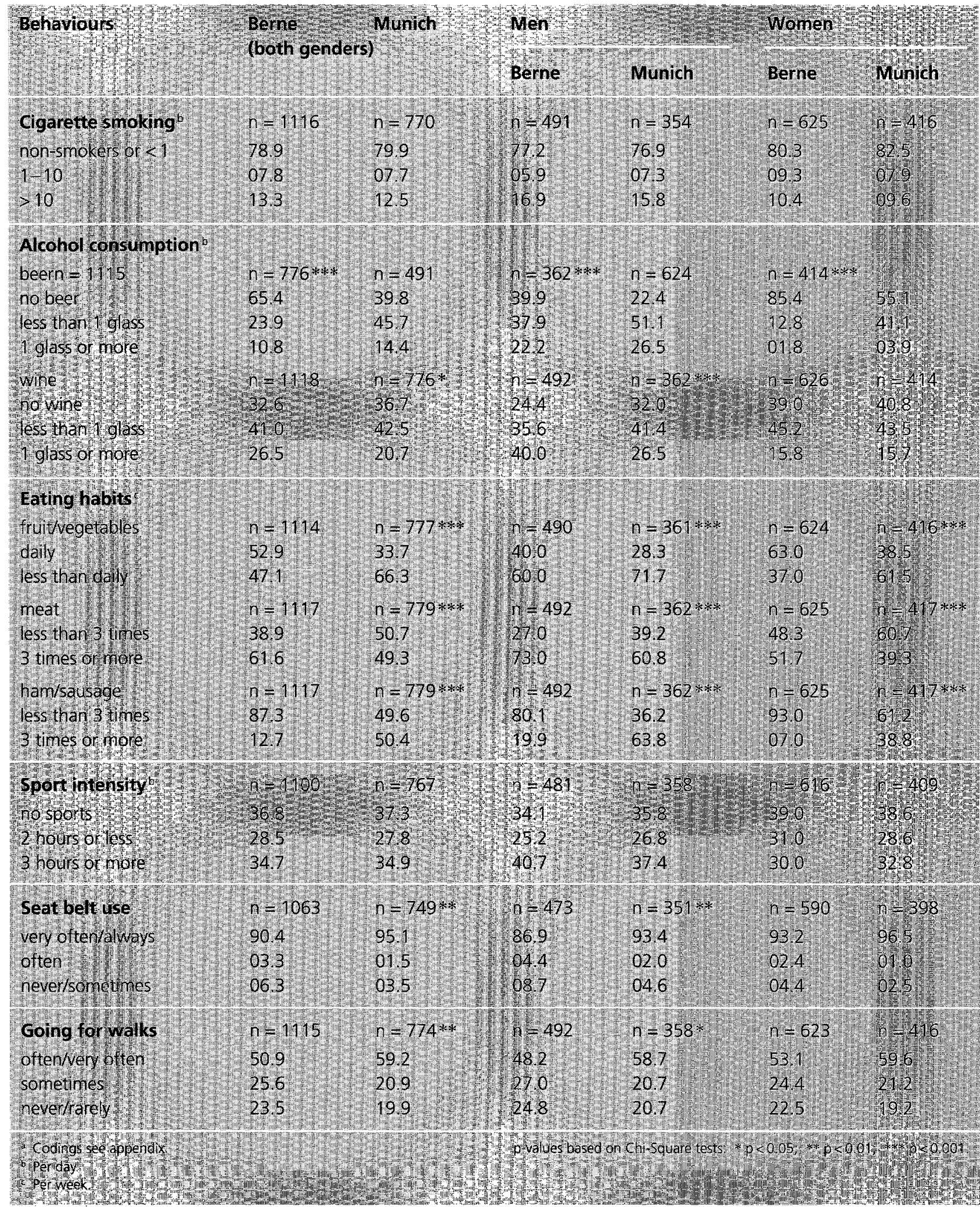

Table 1. Health related behaviours in the BMLP. Presented are row percentages first for the two city samples and second for men and women separately. Significance tests apply respectively to culture differences between the two city samples and to cultural differences within each gender group ${ }^{a}$. 


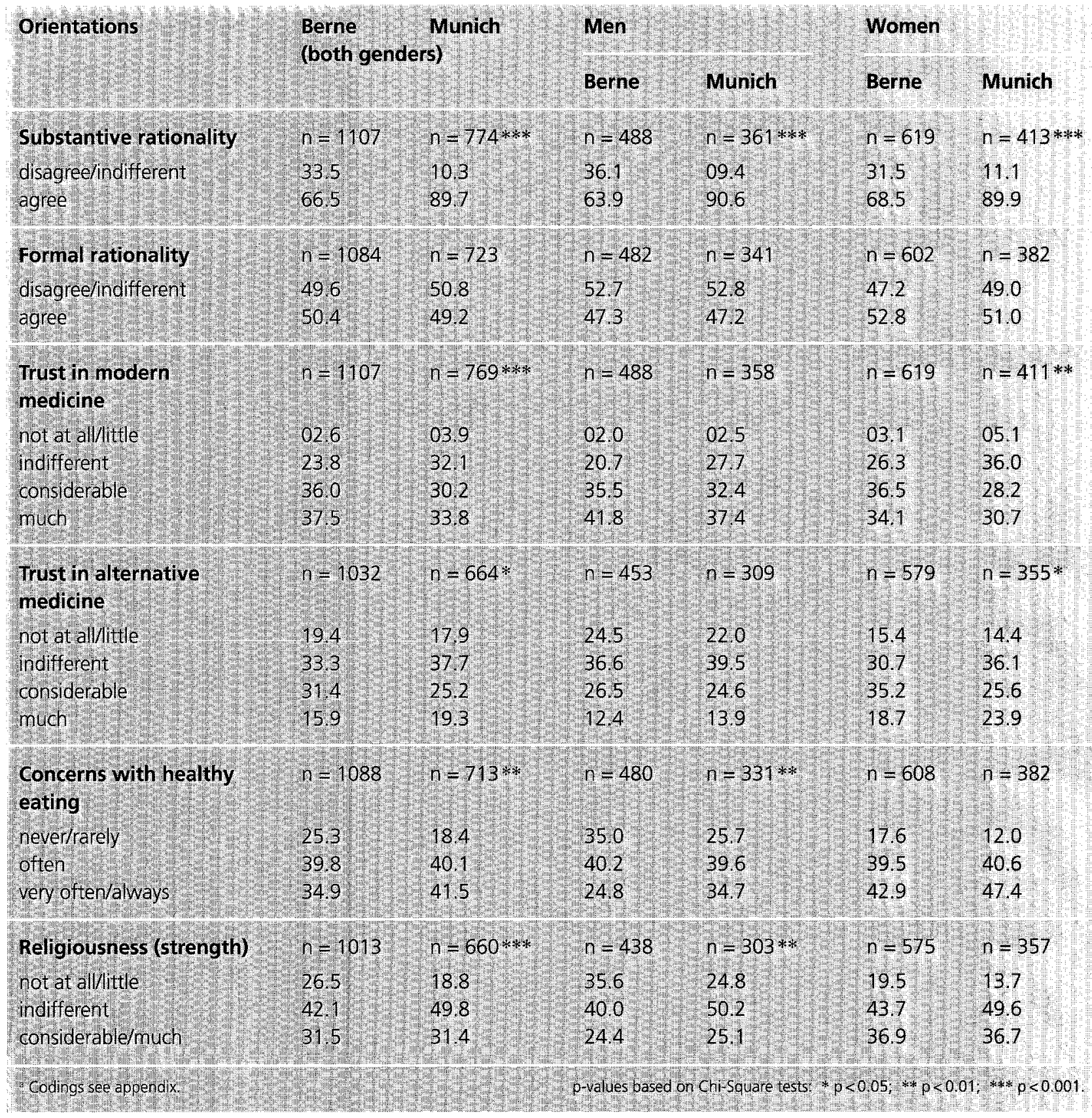

Table 2. Health related orientations in the BMLP. Presented are row percentages first for the two city samples and second for men and women separately. Significance tests apply respectively to culture differences between the two city samples and to cultural differences within each gender group ${ }^{a}$.

both samples agreed on substantive reasons for health enhancing behaviours in the general population (see Table 2). Yet, cultural differences were found as answers in Munich showed higher scores on substantive values (Berne: $66.5 \%$, Munich: $89.7 \% ; \mathrm{p}<0.001$ ).
This cultural difference is found among men and women. Agreement scores for formal rationality were generally lower than for substantive rationality (Berne: $50.4 \%$, Munich: $49.2 \%$ ) and yielded no significant cultural differences.
Trust in modern medicine appears stronger in Berne $(37.5 \%$ in the highest category) than in Munich $(33.8 \%, \mathrm{p}<0.001)$. However, this difference holds only among women $(\mathrm{p}<0.01)$. Strong trust in alternative medicine is overall less frequent when compared to mo- 
dern medicine and more prevalent in the Munich compared to the Bernese sample: $19.3 \%$ in Munich but only $15.9 \%$ of respondents in Berne report much trust in alternative medicine $(p<0.05)$. As indicated by the gender separating statistics table this slight cultural difference is to be attributed to a significant variation among women only $(\mathrm{p}<0.05)$.

Concerns with healthy eating are stronger among Munich than among Bernese respondents ( $\mathrm{p}<$ 0.01 ). Gender specific analysis reveals that this cultural difference is mostly due to higher scores among Munich men, when compared to Bernese men $(\mathrm{p}<0.01)$. Women in Munich and Berne pay more attention to their diet than their male counterparts and do so to a similar degree in both cultures (always/very often: Bernese women $42.9 \%$, Munich women $47.4 \%$ ).

On average, reported strength of religious beliefs is lower in our Bernese sample $(\mathrm{p}<0.001)$. This difference is mostly due to a higher proportion reporting no or almost no religious beliefs in Berne (Berne: 26.5\%, Munich: $18.8 \%)$. There is no such cultural variation among women. The statistical difference is therefore due to variations among men only $(\mathrm{p}<0.01)$.

As the summary table (Table 5) shows, health lifestyle orientations vary considerably between the two samples, with formal rationality in health behaviour being the only exception. Cultural differences in the perception of medicine show a gender specific effect, as only women differ significantly between the two samples while men in Berne and Munich report highly similar orientations towards modern and alternative medicine. On the other side, for men there is a significant cultural difference in their orientation towards healthy eating and religiousness while in this respect women in Berne and Munich appear to be more alike.

\section{Resources}

Monthly household income is higher in our Bernese sample, for women as well as for men $(p<0.001)$. Even after taking into account that standard costs of living might be about $15-20 \%$ higher in Berne compared to Munich, the difference is still considerable. However, with respect to our second measure of financial resources (savings, property etc.) Munich respondents report more privileged property conditions $(p<0.001)$. This difference holds again for both genders. Education as measured by highest educational degree earned is higher among Bernese respondents ( $p<0.001)$. Occupational prestige is similar in both samples; yet, among women occupational prestige is higher in Munich than in Berne $(\mathrm{p}<0.001)$. Quantity of leisure time was assessed based on respondent's perception of subjective sufficiency. This measure is used here as an approximation of leisure time free of personal obligations. Time in that sense can be considered as a resource e.g. for engaging in health enhancing behaviours. Applying three categories "(much) too little", "just enough" or "too much" leisure time, we find no significant differences between Bernese and Munich respondents. Gender specific analysis shows, however, more favorable scores for this health lifestyle resource among Bernese women compared to their Munich counterparts $(\mathrm{p}<0.05)$. Social support as an indicator for social resources for health and health behaviours was assessed in a simple form by asking the number of persons (within and outside the family) one can rely on in difficult situations. The proportion of respondents who reported more than one such support person, was significantly higher in the Bernese sample $(\mathrm{p}<0.001)$. About one fifth among Munich respondents reported none or one support person within the family (in Berne $10 \%$ ).
Some $40 \%$ in Munich had less than two persons outside their family (in Berne $20 \%$ ). This cultural difference is found in both gender groups.

An overview of the observed differences in the distribution of resources is provided in Table 5 . Overall, respondents in the Bernese sample appear better off with respect to most of the social and economic resources examined here. Bernese respondents report better conditions with respect to income, education and social support. Furthermore, women in Berne report a higher degree of satisfaction with the amount of leisure time, while Munich women show higher occupational prestige scores when compared to their Bernese counterparts. Except for the property and for the prestige variables Bernese women report more favourable social resources relevant for health lifestyles than Munich women.

\section{Lifestyle change factors}

Table 4 presents comparative findings on such variables that are considered as triggers or modifying factors for health lifestyle changes. Based on a standardised list of 18 life events ${ }^{13}$ a comparison between the two city samples shows a similar number of events reported for the last 12 months. In both city samples only about $25 \%$ of respondents reported none of such events. We found no significant differences among women in both cities. Munich men, however, showed higher incidence rates for life events than their Bernese counterparts $(p<0.05)$. More frequent events included retirement, financial crisis, illness or accident of partner.

Three dimensions of Health Locus of Control (internal, external control of health by medical experts, fate) were measured utilising a standardised HLC scale ${ }^{14}$. Answer scores were categorised for each dimension into low, medium/in- 


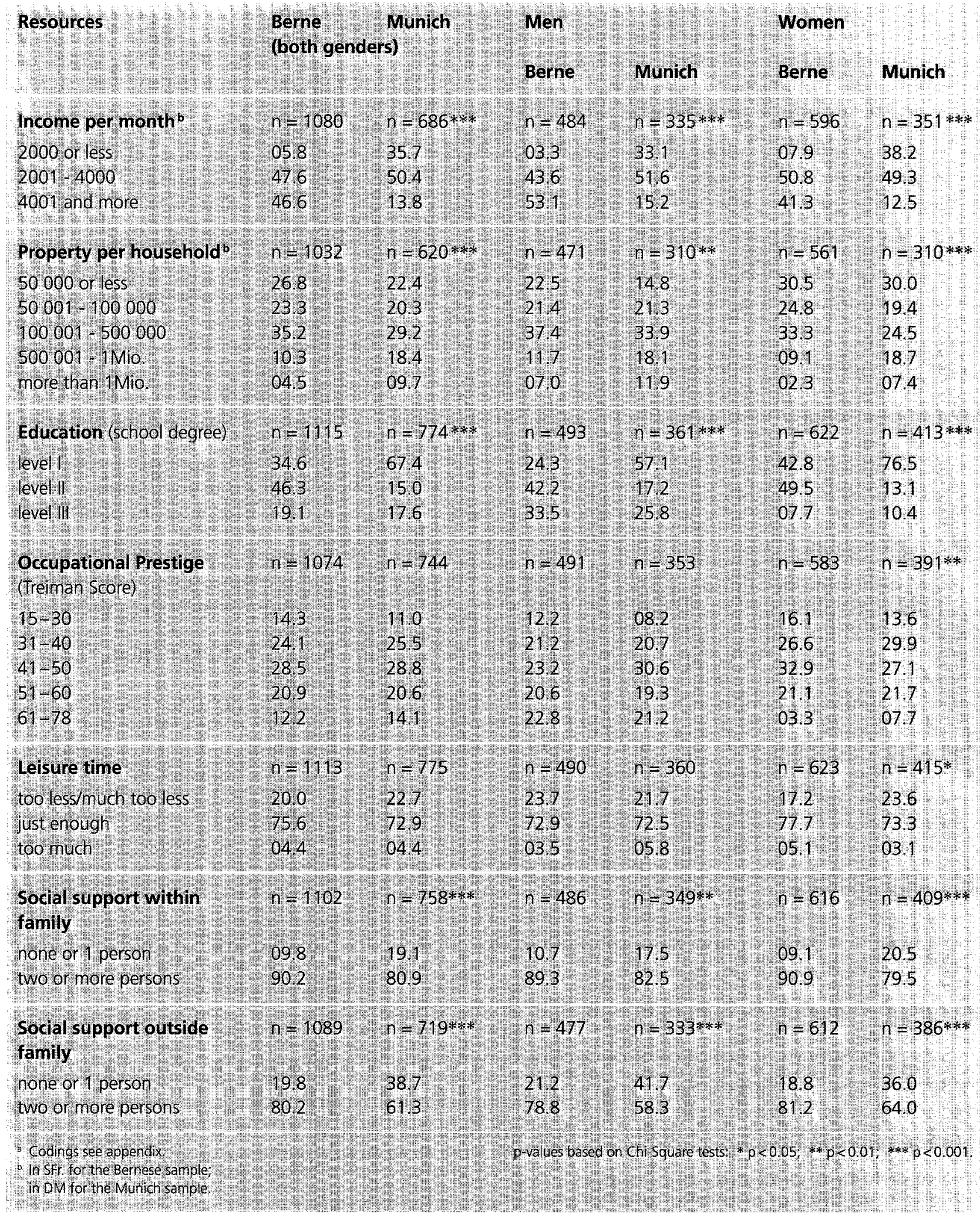

Table 3. Social resources in the BMLP. Presented are row percentages first for the two city samples and second for men and women separately. Significance tests apply respectively to culture differences between the two city samples and to cultural differences within each gender group ${ }^{2}$. 


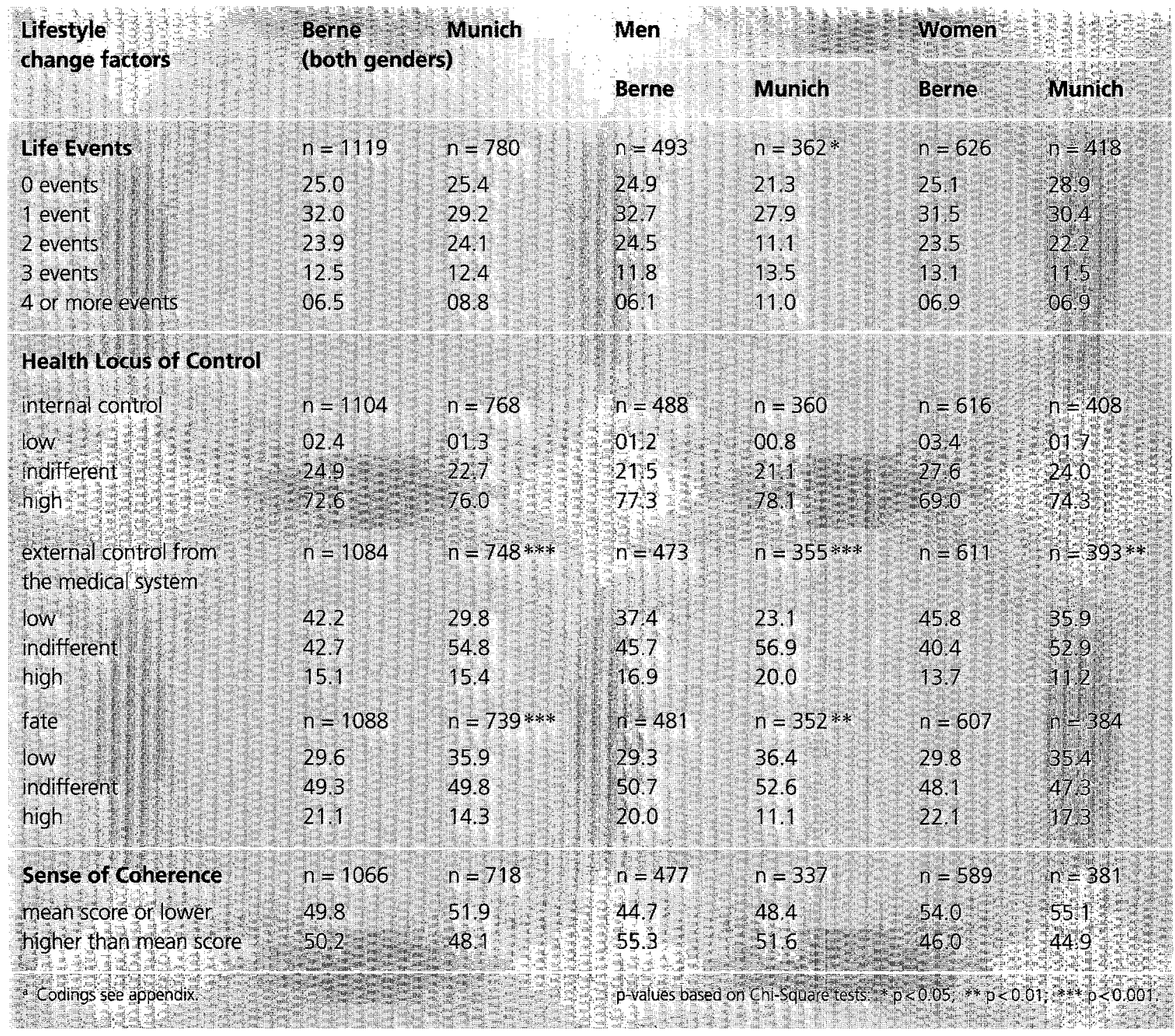

Table 4. Lifestyle change factors in the BMLP. Presented are row percentages first for the two city samples and second for men and women separately. Significance tests apply respectively to culture differences between the two city samples and to cultural differences within each gender group ${ }^{a}$.

different and high. A majority of male and female respondents in both cities show a high score on internal control (Berne 72.6\%, Munich $76 \%$ ). In contrast, only some $15 \%$ (in Berne and in Munich) yielded high scores on external control. Low external medical control was significantly less frequent among Munich respondents in general $(\mathrm{p}<0.001)$ as well as in both gender groups. Significantly more Bernese re- spondents believe strongly in fate affecting their health $(\mathrm{p}<0.001)$. Yet, gender specific analysis reveals that this cultural effect is found among men only. While there is no significant difference between Bernese and Munich women, Bernese men are more likely to have a stronger fate attitude $(20 \%)$ than their male counterparts in Munich $(11.1 \%, \mathrm{p}<0.01)$. Sense of Coherence was measured by applying the 13 item short ver- sion proposed by Antonovsky ${ }^{15}$. Overall, scores ranged from 25 to 64 points and were on average high (mean scores Munich: 50.0; Berne: 51.3). No cultural difference for either the sample as a whole or within each gender group was observed for the degree of SOC.

Patterns of health behaviours

The lifestyle approach draws attention to the patterning of behav- 


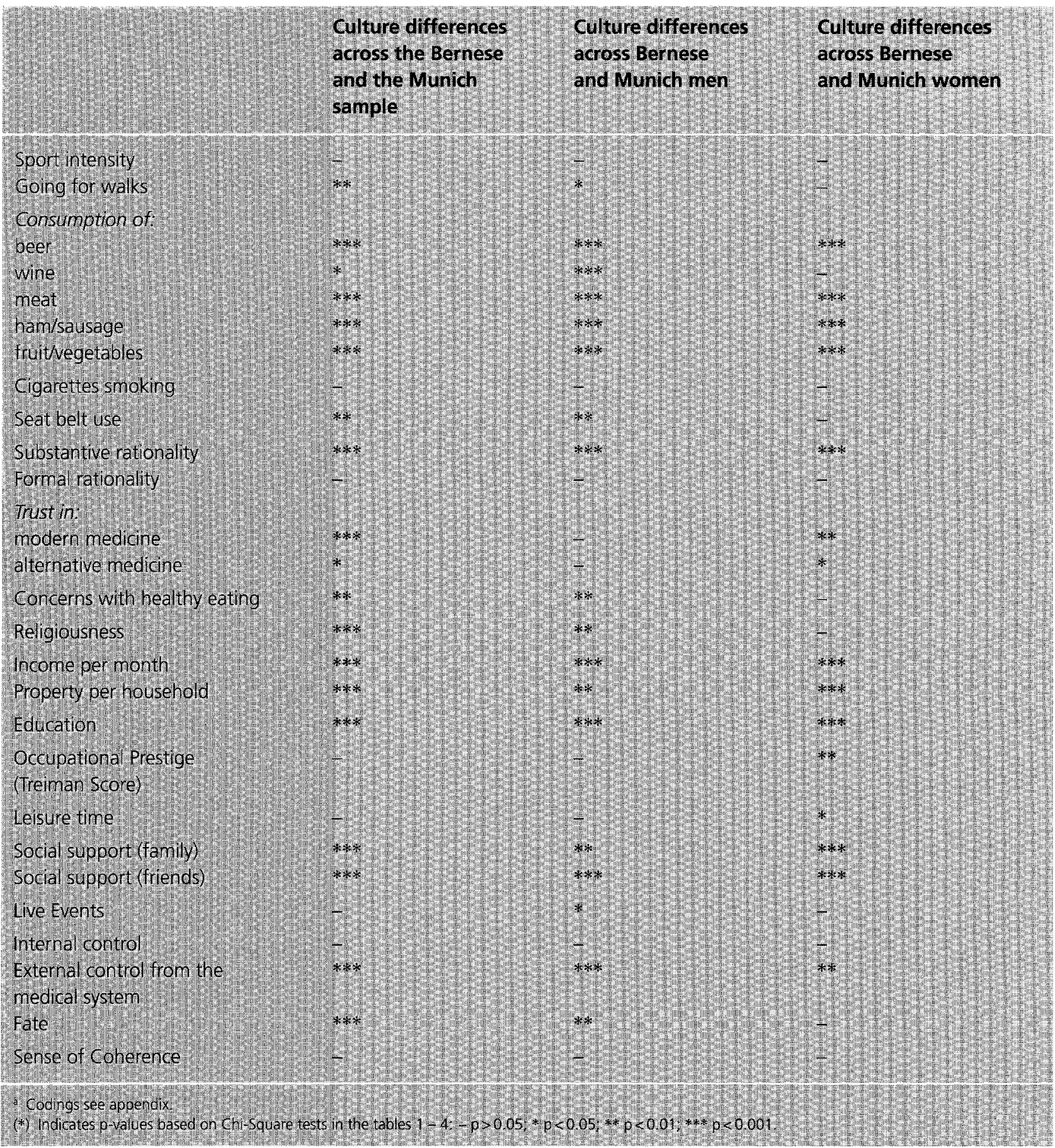

Table 5. Overview of cultural variations in health lifestyle variables observed in Tables $1-4^{a}$.

ioural, social-psychological and social-structural factors. A first step towards the study of such patterns is the exploration of bi-variate relationships among selected health lifestyle items. Tables $6,6 \mathrm{a}, 6 \mathrm{~b}$ pre- sent results from correlation analysis of health lifestyle behaviours in the BMLP (for the two city samples and gender subgroups). Variables were coded with higher scores indicating positive behav- iours from a health promoting perspective. Only coefficients $r \geq 0.10$ (cut-off level) are considered here. A first general inspection of Table 6 reveals that for almost all correlations above the cut-off level 


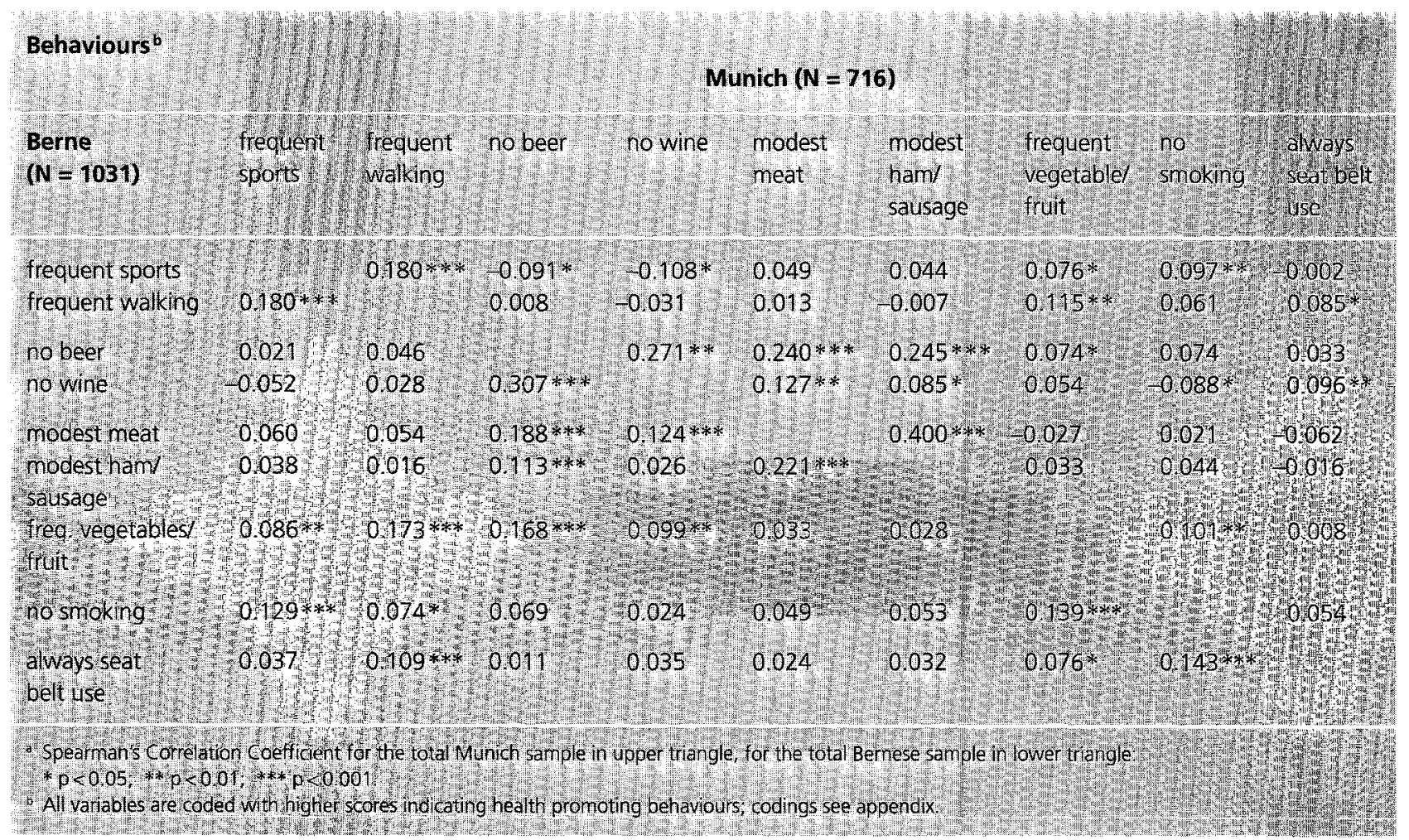

Table 6. Correlation coefficients for health related behaviours ${ }^{a}$.

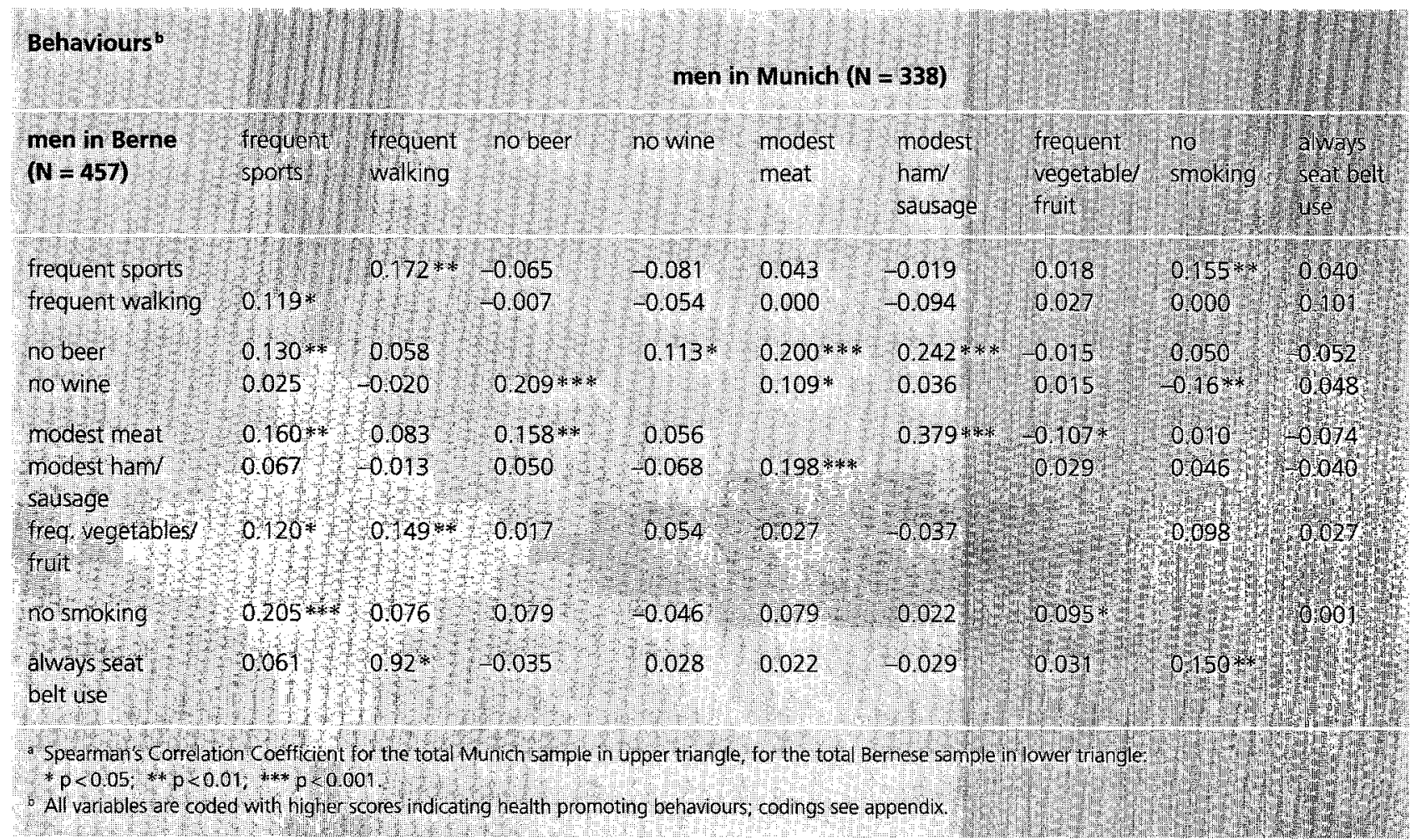

Table 6a. Correlation coefficients for health related behaviours (Berne-Munich men) . 


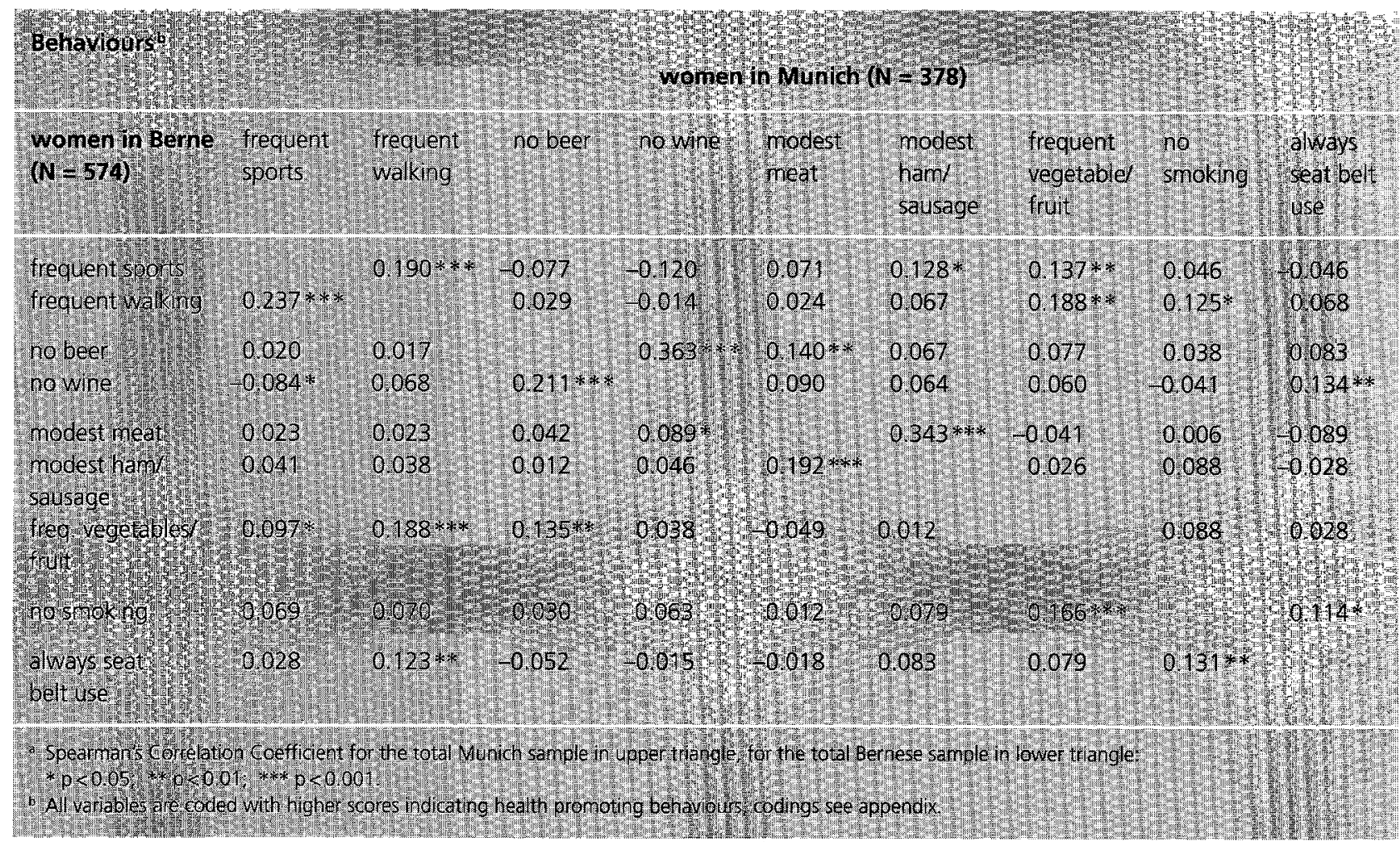

Table $6 \boldsymbol{b}$. Correlation coefficients for health related behaviours (Berne-Munich women) ${ }^{\text {. }}$

statistical associations are positive indicating considerable consistency among those behaviours in Berne and in Munich. Correlation coefficients are on average modest to low. This is in line with findings from earlier studies and was to be expected, given the exploratory character of the study. All bivariate associations above $r \geq 0.10$ are statistically significant at least at the $\mathrm{p} \leq 0.05$ level. With one exception (seat belt use in Munich) all health behaviour variables have a significant correlation with at least one other health behaviour variable.

Our further inspection of correlation Tables 6 to $6 \mathrm{~b}$ concentrates first on associations between different forms of the same kinds of health behaviours, e.g. between beer and wine drinking as two forms of the same kind of health behaviour i.e. alcohol consumption. Two other kinds of health behaviours to be explored in that respect are eating behaviours and physical activities. As for the later, a positive correlation between our variables Sport and Walks indicate that respondents who practice either of the two are also more likely to engage in the other. This relationship is found in both city samples and holds for both genders.

Findings on eating behaviours indicate considerable associations between the variables Meat and Ham. Respective results show that among men and women those who consume less meat are also more likely to eat less ham and sausage. This association is considerably stronger among Munich respondents. Among Munich men, we also observe a link between reduced meat consumption and eating less foods rich in vitamin $\mathrm{C}$.

Significant correlations between our variables Wine and Beer indicate a considerable, and partly expected consistency in these two forms of alcohol (none) consumption. They suggest that those who don't drink either of the two are also more likely to abstain from the other. Among men the association between no wine and no beer drinking is considerably stronger in Berne. Among women this link appears stronger in Munich than in Berne. Consecutive crosstables analyses (not shown) revealed that among Bernese men $63 \%$ of those who don't drink wine also drink no beer, while among those who don't drink beer only $38 \%$ also consume no wine. This means that among Bernese men those who don't drink beer are more than 50 percent likely to abstain also from wine drinking, whereas this relationship does not show in the other direction. Among Bernese women, no wine consumption is again highly indicative also for drinking no beer, as $94 \%$ of those who drink no wine also don't drink beer. However, only $43 \%$ of those who drink no 
beer also drink no wine. The same relationship is observed among Munich female respondents for whom no wine consumption is again more predictive for no beer drinking as the other way around. Only for Munich men no beer consumption appears a relatively stronger indicator, as among them $57 \%$ of those who report no beer consumption also don't drink wine, while only $40 \%$ of those with no wine consumption also drink no beer.

Table 6-6b also allow a first exploration of bi-variate relationships between different kinds of behaviours. Less alcohol consumption is positively correlated with more healthy eating. While this association appears stronger for beer compared to wine drinking, there are considerable cultural and gender differences. For example, the positive association between (less) beer and (less) meat consumption is found in Munich for both gender groups, in Berne only for men. Only for Bernese women less/no beer consumption is positively correlated with eating more foods rich in vitamin C. Nonsmoking shows positive associations with increased sport participation (only men) and with seat belt use (except Munich men). These and other associations warrant further investigation.

\section{Conclusions}

This paper has introduced the Berne-Munich Lifestyle Panel (BMLP), its theoretical background, its basic empirical model and first comparative results. Overall such results show cross-cultural similarities as well as considerable variations in the distribution of health lifestyle behaviours, orientations and social resources. For health behaviours we have explored relationships between different kinds of health behaviours and between different forms of the same kinds of behaviours. As for the later, the results on wine and beer drinking clearly demonstrate the importance of culture and gender specific investigations when studying patterns of alcohol consumption. We found the links between drinking patterns considerably different in the four subgroups of our sample. Beer consumption appears the standard alcohol drink for Munich men only. In this subgroup abstaining from beer is more predictive for not drinking other sorts of alcohol. Among Munich women and for the Bernese population this "reference" effect is not found for beer but for wine. Also, across the two cultures, this reference effect appears stronger for women compared to men.

The findings on the associations between different kinds of health behaviours like that between healthy eating, non-smoking and physical activity generally support the idea of a patterning of health behaviours and add to the increasing empirical evidence in this field of health behaviour research ${ }^{17-19}$. As the present results are based on analyses of bi-variate associations, they still warrant further investigations, applying more sophisticated statistical methods. As previously demonstrated, Cluster- and Correspondence analysis can be applied to study the complex structures of health lifestyles ${ }^{5,20}$. Logistic regression modelling has shown to be an appropriate approach in estimating the effects of one lifestyle dimension on the others ${ }^{6}$. Also, new approaches in graphical modelling appear applicable for future analysis of multi-dimensional health lifestyle data.

In general, the empirical findings presented here stress the importance of context effects on health behaviours. The role of culture and gender conditions on the prevalence and the patterning of health relevant behaviours is strongly underlined by the present results. However, as explained in the theory part, health behaviours are only one aspect of broader health lifestyles. Consequently, more comprehensive models need to be developed. Sociological theory has provided guidelines for such endeavours and suggests that future empirical investigations should concentrate on the links between health behaviour patterns, health orientations and social resources. 


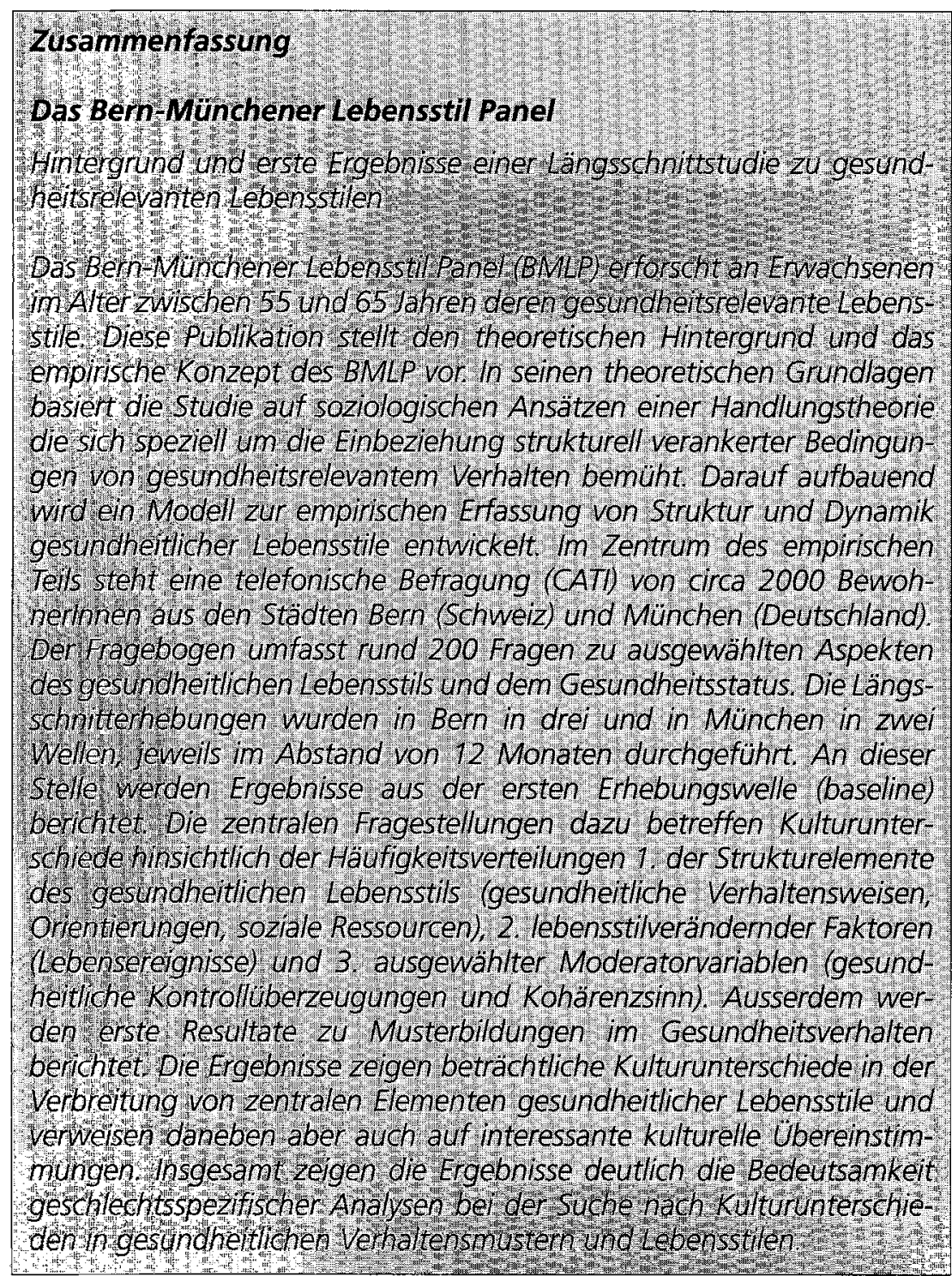

\section{References}

1 Blaxter $M$. Health and Lifestyles. London: Tavistock, 1990.

2 Cockerham WC, Rütten A, Abel T. Conceptualizing Contemporary Health Lifestyles: Moving beyond Weber. The Sociological Quarterly 1997; 38:321-342.

3 Abel T. Gesundheitsverhaltensforschung und Public Health: Paradigmatische Anforderungen und ihre Umsetzung am Beispiel gesundheitsrelevanter Lebensstile. In:
6 Abel T, Kohlmann T. Health lifestyles: a comparative approach to the culture of health concept. In: Lüschen G, Cockerham WC, Kunz $\mathrm{G}$, eds. Health and illness in America and Germany. München: Oldenbourg, 1989: 71-82.

7 Uitenbroek $D G$. Health lifestyle behaviour and sociodemographic characteristics. A study of Varna, Glasgow and Edinburgh. Soc Sci Med 1995; 43:376-377.

8 Cockerham WC, Abel T, Lüschen $G$. Max Weber, formal rationality and health lifestyles. The Sociological Quarterly 1993; 34:413-425.

9 Noack RH. Gesundheit: Medizinische, psychologische und soziologische Konzepte. In: Gawatz R, Novak P, eds. Soziale Konstruktionen von Gesundheit. Ulm: Universitätsverlag, 1993: 13-32.

10 Buckley W. Modern systems research for the behavioral scientist. Chicago: Aldine Publ. Company, 1968.

11 Abel T, Karvonen $S$, Weitkunat $R$. Zur Bedeutung von Wert- und Zweckrationalität für gesundheitsrelevanten Sport und körperliche Aktivität. Eine explorative Analyse. In: Rütten A, ed. Sport und Public Health. Stuttgart: Verlag Stefanie Nagelschmid, 1998: 241258.

12 Treiman DJ. Occupational Prestige in comparative perspective. New York: Academic Press, 1977.

13 Brugha T, Bebbington P, Tennant C, Hurry $J$. The list of threatening experiences: a subset of 12 life event categories with considerable long-term contextual threat. Psychological Medicine 1985; 15:189194.

14 Wallston KA, Wallston BS, DeVellis $R$. Development of the multidimensional Health Locus of Control (MHLC) scales. Health-EducationMonographs 1978; 6:160-170.

15 Antonovsky $A$. Health, stress, and coping: new perspectives on mental and physical well-being. San Francisco: Jossey-Bass, 1979.

16 Abel T, Kohlmann T, Noack RH. Eine deutsche Übersetzung des 


\section{Résumé}

Cadre gènéral et premiers résultats d'une étúde longitudinale sur fes interactions entre modes de vie et santé:

\section{Le Bern-Münchener Lebensstil Panel (BMLP)}

Le BMLP est une étude sur les modes de vie de la classe d'âge des 55-65 ans et sur feurs implications pour la sante. L'article publié ici décrit l'approche théorique et la méthodologie appliquée dans le cadre de cette étude. Au plan théorique, le BMLP se base sur différents módèles d'analyse sociologique développés autour de l'axiome central selon lequel les comportements en matiere de santé dépendent étroitement des conditions structurelles envionnantes. Au plah emprique, Ja méthodologie développee pour discerner ces structures et, par conséquent, la dynamique qui sous-tend les modes de vie ayant un impact sur la santé a donc été élaborée dans le droit-fil de ces fondements theoriques. La partie empirique de l'étude repose sur une enquête par téléphone (CATI), réalisée aupres de 2000 personnes habitant les villes de Berne (Suisse) et de Münich (Allemagne), Le questionnaire rédigé à cet effet comprend plus de 200 questions, portant sur des indicateurs precis, deffnis otavance comme revelateurs du mode de vie et de létat de santé de ces personnes. Les donnees collectees lors de cefte etude longitudinale résultent de trois séries d'enquête a Berne et de deux séries à Munich; Iintervalle entre deux campagnes telephoniques etant a chaque fois d'un an. Les résultats présentés ici sont ceux de la premiere campagne téléphonique (baseline). Les réflexions et les questionnements auxquels is donnent fieu tournent surtout autour des dimensions culturelles qui sous-tendent les différences relevées dans la fréquence d'apparition: 1. des aspects structurels ayant une incidence sur les modes de vie en matere de santé fomportements, attitudes, ressources sociales) 2. des facteurs de changement (événements de vie) et 3 . des variables considerées comme éléments de ponderation (croyances fondamentales en matiere de sante et sens de la cohérence). De plus, ces résultats permettent d'esquisser une première typotogie des comportements en matière de sante. Les résultats obtenus dans le cadre du BMLP mettent en lumiere des differences culturelles tres nettes en ce qui concerne certains éléments déterminants pour le mode de vie et la santé, tout en revelant également des convergences fort interessantes. Dans l'ensemble, rétude confirme aussi la pertinence d'une analyse différenciée selon les sexes, dès fors qu' on cherche à mieux appréhender les facteürs socioculturels explicatifs des comportements en matière de santê ef des styles de vie d'une population donnee.

SOC-13. Arbeitspapier der Abteilung für Gesundheitsforschung des Instituts für Sozial- und Präventivmedizin der Universität Bern. 1995 (unveröffentl.).

17 Dean $K$. Self-care components of lifestyles. The importance of gender, attitudes, and the social situation. Soc Sci Med 1989; 29:137152.
18 Prättälä RS, Laaksonen $M T$, Rahkonen $O J$. Smoking and unhealthy food habits. How stable is this association? European Journal of Public Health 1998; 8:28-33.

19 Steptoe A, Wardle J, Fuller R, et al. Leisure-time physical exercise. Prevalence, attitudinal correlates, and behavioral correlates among young Europeans from 21 countries. Pre- ventive Medicine 1997; 26:845854.

20 Lüschen $G$, Niemann $S$. Health life-style and social stratification. In: Lüschen G, Cockerham WC, v.d. Zee J, et al., eds. Health systems in the European Union, diversity, convergence, and integration. München: Oldenbourg Verlag, 1995: 101-112.

\section{Address for correspondence}

Prof. Dr. Thomas Abel Ph.D.

Unit for Health Research

ISPM

University of Berne

Niesenweg 6

CH-3012 Berne

Fax: ++41316313430 


\section{Appendix}

Items Variables

\section{Behaviours}

1 Sport and physical exercise hours per week

1 Seat belt use when driving or riding a car

1 Daily consumption of beer

1 Daily consumption of wine

1 Consumption of ham/sausage in a regular week

1 Consumption of meat in a regular week

3 Consumption of vegetables/salad; fruit; potatoes in a regular week

$1 \quad$ Number of cigarettes per day

1 Seat belt use when driving or riding a car

\section{Orientations}

$3 / 2^{7} \quad$ Substantive Rationality in health behaviour

5 Formal Rationality in health behaviour

2 How much do you trust in conventional/modern medicine; how much in alternative/natural medicine?

8 How much do you pay attention that you eat not: too much; too sweet; too fatty; too salty; that you eat: enough vitamins; varied; regularly; whole meal food

1 Strength of religious beliefs

\section{Resources}

$1 \quad$ Household income per month adjusted by number of househoid members

1 Household property

$1 \quad$ Highest educational degree

8 Occupation-Prestige based on the Treiman-Scale

1 General Self estimate about the degree of time at one's own disposition (leisure time)

2 Number of people within respectively outside the family that one can rely on in difficult situations

\section{Life change factors}

18 List of life events

$9 \quad$ Health Locus of Control: internal, external (medical system), fate (3 Items each) Sense of Coherence (SOC 13 Tele)

\author{
$0=$ no sports, $1=1-2$ hours, $2=3$ hours or more 2 \\ $1=$ never/rarely, 2 = often, $3=$ very often/always ${ }^{3}$ \\ $3=$ no beer, no alcohol at all, 2 = less than 1 glass ${ }^{4}$, \\ $1=1$ glass or more \\ $3=$ no wine, no alcohol at all, 2 = less than 1 glass, \\ $1=1$ glass or more \\ $1=$ less than 3 times per week, \\ $0=3$ times per week or more \\ $1=$ less than 3 times per week, \\ $0=3$ times per week or more \\ $51=$ daily consumption of at least two of these three \\ main sources of vitamin $C, 0=$ no daily consumption \\ of at least two of these three main sources of vitamin $C$ \\ $3=$ non smokers, smokers but less than 1 cigarette per day, \\ $2=1-10$ cigarettes, $1=$ more than 10 cigarettes \\ $1=$ never/rarely, 2 = often, 3 = very often/always ${ }^{6}$ \\ ${ }^{81}=$ strongly disagree/disagree/partly agree, \\ 2 = strongly agree/agree \\ ${ }^{71}$ = strongly disagree/disagree/partly agree, \\ 2 = strongly agree/agree \\ for each item: 1 = little/not at all, 2 = partly yes and no, \\ $3=$ considerable, $4=$ very strongly \\ ${ }^{9} 1=$ never/rarely, 2 = often, 3 = very often/always \\ ${ }^{10} 1$ = none/low, 2 = somewhat, $3=$ considerable/much; \\ $111=0-2000,2=2001-4000,3=4001$ or more \\ $81=$ up to $50000,2=50001-100000$, \\ $3=100001-500000,4=500001-1$ Mio., \\ $5=$ more than $1 \mathrm{Mio}$ \\ $121=$ level I, $2=$ level $I 1,3=$ level III \\ $1=14-30,2=31-40,3=41-50,4=51-60$, \\ $5=61-78$ \\ $1=$ none or much too little, 2 = just enough, \\ $3=$ too much \\ $1=0-1$ person, $2=$ more than 1 person
}

\footnotetext{
1 If there is no other annotation missing values are "no answer" and "answer refused".

2 The categories have been built on basis of the bernese peoples mean ( $M=2,6$ hours).

${ }^{3}$ Missing: not driving a car (in Berne 56 cases, in Munich 30 cases).

4 Standard unit in Berne: 1 glass = $0.3 \mathrm{dl}$; standard unit in Munich: 1 glass $=0.5 \mathrm{dl}$.

5 Each item coded from 1 (not at all) to 5 (daily); sumscore recoded in 2 categories.

6 Missing: not driving a car (in Berne 56 cases, in Munich 30 cases).

7 Reliability analyses resulted in 3 items for Berne and 2 for Munich.

8 Each item coded from 1 (strongly disagree) to 5 (strongly agree); sumscore recoded in 2 categories.

9 Each item coded from 1 (never) to 5 (always); sumscore recoded in 3 categories.

${ }^{10}$ Missing: all respondents with other confessions than catholic, protestant/reformiert or evangelist (in Berne 100 cases, in Munich 116 cases).

11 In Berne in SFr.; in Munich in DM; $1 \mathrm{DM}=0.83 \mathrm{SFr}$.

12 Level I: no degree, degrees from obligatory schools (up to 9 years of schooling); level II: degree from advanced schooling (up to 13 years); level III: high education such as University degree.

13 Each item coded 0 (no event) or 1 (event); sumscore recoded in 4 categories.

14 The maximum of events was 8 in Berne and 9 in Munich respectively.

15 Each item coded from 1 (strongly disagree) to 5 (strongly agree); sumscore recoded in 3 categories.

16 Mean in Berne 51, mean in Munich 50
} 